\title{
MULTISCALE OR NO MULTISCALE ANALYSIS FOR MASS TRANSFER IN A MICRO/NANOCHANNEL?
}

\author{
Zhipeng Tang and Yongbin Zhang ${ }^{\text {b* }}$ \\ ${ }^{a}$ College of Mechanical Engineering, Changzhou Vocational Institute of Mechatronic Technology \\ ${ }^{b}$ College of Mechanical Engineering, Changzhou University, Changzhou, 213164, Jiangsu, China
}

\begin{abstract}
The mass flow rate through a micro/nano channel is calculated by a multiscale analysis when the thickness of the adsorbed layer on the channel wall is comparable to the channel height and the interfacial slippage on the adsorbed layer-wall surface interface occurs or not. The calculation is compared with that from conventional continuum flow theory. It is found that when the ratio $\lambda_{\mathrm{bf}}$ of the thickness of the adsorbed layer to the film thickness of the intermediate continuum fluid is no more than 0.02 , normally the effect of the adsorbed layer is negligible and a (no multiscale) continuum flow theory can calculate the flow rate through the channel in spite of the fluid-wall interaction, whenever the interfacial slippage occurs or not. Both the increase of $\lambda_{\mathrm{bf}}$ and the increase of the interfacial slippage enlarges the proportion of the adsorbed layer flow rate to the total flow rate through the channel. For $\lambda_{\mathrm{bf}}$ larger than the critical value which is dependent on both the fluid-wall interaction and the interfacial slippage, a multiscale analysis is normally required for calculating the flow rate through the channel by incorporating the adsorbed layer effect. However, for a great interfacial slippage such as for the dimensionless power loss $A$ on the channel for driving the interfacial slippage over 100, a continuum flow theory may be sufficient in the entire range of $\lambda_{\mathrm{bf}}$ for calculating the flow rate through the channel by considering the interfacial slippage and ignoring the existence of the adsorbed layer, when the fluid-wall interaction is medium-level or strong.
\end{abstract}

Keywords: Flow; Mass transfer; Micro/nano channel; Multiscale; Slippage

\section{INTRODUCTION}

Macrochannel flows can be well described by continuum flow theories neglecting the adsorbed layer on the channel wall, the thickness of which may be on the $1 \mathrm{~nm}$ scale and far lower than the channel height (Cho, 1982; Kawamura et al., 1986; Lemenand and Peerhossaini, 2002; Vallikivi et al., 2011). Nanochannel flows with the channel height on the $1 \mathrm{~nm}$ scale should normally be described by non-continuum flow theories which incorporate the discontinuity and inhomogeneity of the fluid across the channel height (Gee et al., 1990; Jabbarzadeh et al., 1997; Joseph et al., 2006; Heinbuch and Fischer, 1989; Thompson and Robbins, 1990; Zhang, 2016). There are the channel flows with the channel size scales ranging between and $1 \mathrm{~nm}$ which should be described by the multiscale scheme incorporating both the flow of the non-continuum adsorbed layer and the flow of the continuum fluid intermediate between the two boundary layers. A lot of multiscale schemes have been proposed for such channel flows by using molecular dynamics simulation to simulate the adsorbed layer flow and using continuum flow theories to model the intermediate continuum fluid flow (Atkas and Aluru, 2002; Liu et al., 2007; Nie et al., 2004; Sun et al., 2010; Yang and Zheng, 2010; Yen et al., 2007). Their shortcomings are that they need to take long computational time for a practical long channel flow which is normally unaffordable. Recently, Zhang(2020) proposes a new multiscale scheme for the channel flow by using the flow factor approach model for nanoscale flow to simulate the adsorbed layer flow and using a continuum flow theory to model the intermediate continuum fluid flow.
Three flow equations have been derived respectively for the flows of the two adsorbed layers and the flow of the continuum fluid. This scheme has the advantage of giving fast solutions to a sort of engineering problems. However, it requires to label out the characteristic rheological parameter values of the adsorbed layers, which might need specific work.

In itself, people would more like to calculate the channel flow by using continuum flow theories. When should a multiscale scheme be really required for the channel flow? This is actually a question of significant interest to practice. The present paper aims to address this problem by making multiscale calculations for micro/nano channel flows and comparing these calculations with the conventional continuum theory calculation when the interfacial slippage occurs or not on the adsorbed layer-wall surface interface.

\section{MODELED MICROCHANNEL FLOW}

Figure 1(a) shows the studied multiscale micro/nano channel flow driven by the pressure. Because of its thickness comparable to the channel height, the adsorbed layer on the channel wall may contribute to the flow. The multiscale analysis used in the present study treats the flow in Fig.1(a) as shown in Fig.1(b) (Zhang, 2020). The adsorbed layer flow is essentially non-continuum and described by the flow factor approach model for nanoscale flow (Zhang, 2006); The flow of the intermediate continuum fluid is described by a continuum flow theory. The interfacial slippage may occur or not on the adsorbed layer-wall surface interface depending on the operating condition. No interfacial slippage is assumed on the adsorbed layer-fluid interface. The pressure gradient is assumed 
as negative so that the flow is from the left-hand side to the right-hand side. The used coordinates are also shown in Fig.1(b).

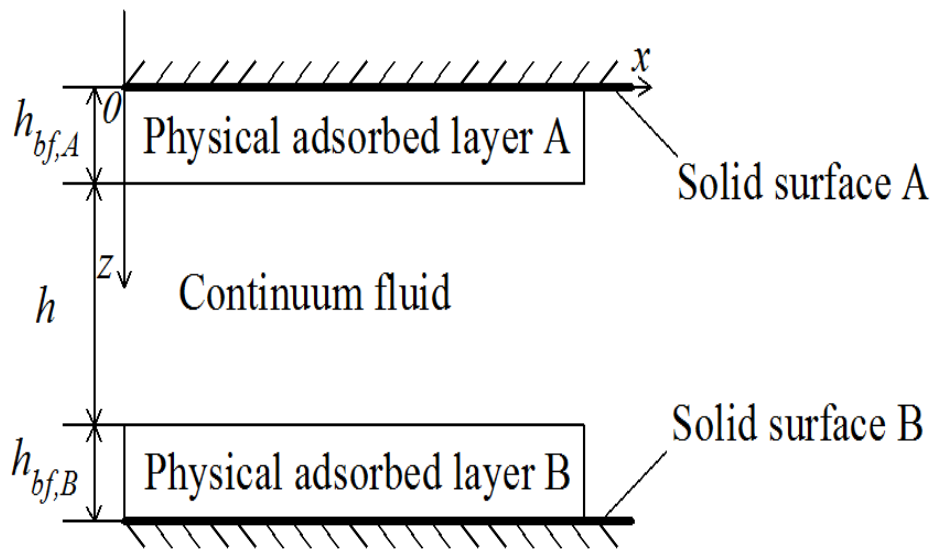

(a)

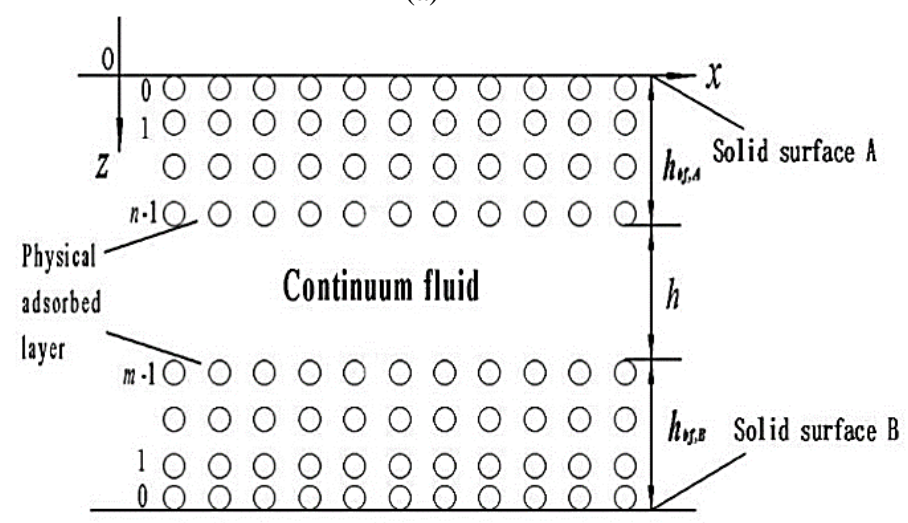

(b)

Fig. 1 Modeled channel flow (Zhang, 2020).

\section{ANALYTICAL RESULTS}

\subsection{Multiscale analysis}

In the present analysis, the adsorbed layers on the two channel walls are assumed as identical. For this case, according to the multiscale analysis developed by Zhang (2020), the volume flow rate per unit channel width of the adsorbed layer is:

$q_{v, b f}=\bar{u} h_{b f}+\left[\frac{F_{1} h_{b f}^{3}}{12 \eta_{b f}^{e f f}}-\frac{h_{b f}^{3}}{2 \eta_{b f}^{e f f}}\left(1+\frac{1}{2 \lambda_{b f}}-\frac{q_{0}-q_{0}^{n}}{q_{0}^{n-1}-q_{0}^{n}} \frac{\Delta_{n-2}}{h_{b f}}\right) \frac{\varepsilon}{1+\frac{\Delta x}{D}} \frac{\partial p}{\partial x}\right.$

where $p$ is the fluid film pressure, $x$ is the coordinate in the flow direction, $\bar{u}$ is the adsorbed molecule velocity on the channel wall, $D$ is the fluid molecule diameter, $\Delta x$ is the separation between the neighboring fluid molecules in the flow direction, $n$ is the equivalent number of the fluid molecules across the adsorbed layer thickness, $h_{b f}$ is the thickness of either of the adsorbed layers on the channel walls, $\lambda_{b f}=h_{b f} / h, \eta_{b f}^{e f f}$ is the effective viscosity of the adsorbed layer, $\eta_{b f}^{\text {eff }}=D h_{b f} /\left[(n-1)\left(D+\Delta_{x}\right)\left(\Delta_{l} / \eta_{\text {line,l }}\right)_{\text {avr }, n-1}\right), q_{0}=\Delta_{j+1} / \Delta_{j}$, $\varepsilon=(2 D I+I I) /\left[h_{b f}(n-1)\left(\Delta_{l} / \eta_{\text {line }, l}\right)_{\text {avr }, n-1}\right]$, and

$F_{1}=\eta_{b f}^{e f f}\left(12 D^{2} \psi+6 D \phi\right) / h_{b f}^{3}$; Here, $h$ is the thickness of the intermediate continuum fluid, $\Delta_{j-1}$ and $\eta_{\text {line }, j-1}$ are the separation and the local viscosity between the $j^{\text {th }}$ and $(j-1)^{\text {th }}$ fluid molecules across the adsorbed layer thickness respectively, $i$ and $j$ are the order numbers of the fluid molecule across the adsorbed layer thickness respectively as shown in Fig.1(b), $I=\sum_{i=1}^{n-1} i\left(\Delta_{l} / \eta_{\text {line }, l}\right)_{\text {avr }, i}, I I=$
$\sum_{i=0}^{n-2}\left[i\left(\Delta_{l} / \eta_{\text {line }, l}\right)_{\text {avr }, i}+(i+1)\left(\Delta_{l} / \eta_{\text {line }, l}\right)_{\text {avr }, i+1}\right] \Delta_{i} \quad, \quad i\left(\Delta_{l} /\right.$

$\left.\eta_{\text {line }, l}\right)_{\text {avr }, i}=\sum_{j=1}^{i} \Delta_{j-1} / \eta_{\text {line }, j-1}, \quad \psi=\sum_{i=1}^{n-1} i\left(l \Delta_{l-1} / \eta_{\text {line }, l-1}\right)_{a v r, i}$,

$\phi=\sum_{i=0}^{n-2}\left[i\left(l \Delta_{l-1} / \eta_{\text {line }, l-1}\right)_{a v r, i}+(i+1)\left(l \Delta_{l-1} / \eta_{\text {line }, l-1}\right)_{a v r, i+1}\right] \Delta_{i}$,

and $i\left(l \Delta_{l-1} / \eta_{l i n e, l-1}\right)_{a v r, i}=\sum_{j=1}^{i} j \Delta_{j-1} / \eta_{l i n e, j-1}$. For the interfacial slippage, $\bar{u}>0$; For no interfacial slippage, $\bar{u}=0$.

The volume flow rate per unit channel width of the intermediate continuum fluid is (Zhang, 2020):

$q_{v, h f}=\bar{u} h+\left\{\frac{h^{3}}{\eta_{b f}^{e f f}}\left[\frac{F_{2} \lambda_{b f}^{2}}{6}-\frac{\lambda_{b f}}{1+\frac{\Delta x}{D}}\left(\frac{1}{2}+\lambda_{b f}-\frac{q_{0}-q_{0}^{n}}{q_{0}^{n-1}-q_{0}^{n}} \frac{\Delta_{n-2}}{h}\right)\right] \frac{h^{3}}{12 \eta}\right\} \frac{\partial p}{\partial x}$

where $\eta$ is the fluid bulk viscosity, and $F_{2}=6 \eta_{b f}^{e f f} D(n-1)\left(l \Delta_{l-1} /\right.$ $\left.\eta_{\text {line,l-1 }}\right)_{\text {avr }, n-1} / h_{b f}^{2}$.

When the interfacial slippage occurs on both of the adsorbed layerwall surface interfaces, the pressure gradient is (Zhang, 2019):

$$
\frac{\partial p}{\partial x}=-\frac{\tau_{s, b-w}}{\frac{1}{2} h+D(n-1)}
$$

where $\tau_{s, b-w}$ is the shear strength of the adsorbed layer-wall surface interface.

The interfacial slipping velocity is equated as (Zhang, 2019):

$\bar{u}=\frac{\Delta P O W\left(1-r_{0}\right)}{2 \tau_{s, b-w^{\Delta l}}}$

where $\Delta l$ is the channel length in the flow direction, $\triangle P O W$ is the power loss on the channel per unit channel width for generating the interfacial slippage, $\triangle P O W=P O W-P O W_{c r}$, and

$r_{0}=\frac{2\left(\frac{q_{0}-q_{0}^{n}}{q_{0}^{n-1}-q_{0}^{n}} \frac{\Delta_{n-2}}{D}+1\right)}{1+2 \lambda_{b f}} \frac{D}{h}$

Here, POW is the power loss per unit channel width on the channel calculated from the multiscale analysis, and $P O W_{c r}$ is the critical power loss per unit channel width on the channel for starting the interfacial slippage calculated from the multiscale analysis.

For estimating the contribution of the adsorbed layer to the flow, the ratio of the total mass flow rate of the two adsorbed layers to the mass flow rate of the continuum fluid is shown as:

$$
\begin{aligned}
& r_{b / h}=\frac{2 \rho_{b f}^{e f f} q_{v, b f}}{\rho q_{v, h f}} \\
&=2 C_{q} \frac{A \lambda_{b f}^{2} \frac{1-r_{0}}{2}-\left\{\frac{F_{1}}{12}-\frac{\varepsilon}{2\left(1+\frac{\Delta x}{D}\right)}\left[1+\frac{1}{2 \lambda_{b f}}-\frac{\left(q_{0}-q_{0}^{n}\right) \Delta_{n-2}}{\left(q_{0}^{n-1}-q_{0}^{n}\right) h_{b f}}\right]\right\} \frac{\lambda_{b f}^{3}}{C_{y}\left[\frac{1}{2}+\frac{1}{h}(n-1)\right]}}{A \lambda_{b f} \frac{1-r_{0}}{2}+\left\{\frac{1}{12}-\frac{1}{C_{y}}\left\{\frac{F_{2} \lambda_{b f}^{2}}{6}-\frac{\lambda_{b f}}{1+\frac{\Delta x}{D}}\left[\frac{1}{2}+\lambda_{b f}-\frac{\left(q_{0}-q_{0}^{n}\right) \Delta_{n-2}}{\left(q_{0}^{n-1}-q_{0}^{n}\right) h}\right]\right\}\right\} \frac{1}{\frac{1}{2}+\frac{D}{h}(n-1)}}
\end{aligned}
$$

where $\rho$ is the fluid bulk density, $\rho_{b f}^{e f f}$ is the average density of the adsorbed layer across the layer thickness, $C_{q}=\rho_{b f}^{\text {eff }} / \rho, C_{y}=\eta_{b f}^{\text {eff }} / \eta$, and $A=\Delta P O W \eta /\left(\tau_{s, b-w}^{2} h_{b f} \Delta l\right)$.

\subsection{Conventional (no multiscale) analysis}

For comparison, the conventional continuum flow analysis for the channel flow is presented in this section. In this analysis, the adsorbed layer is ignored and across the whole channel height is the continuum fluid. In the Poiseuille flow, considering the fluid-wall interfacial 
slippage, the volume flow rate of the continuum fluid per unit channel width through the channel is:

$$
q_{v, \text { conv }}=\bar{u}\left(h+2 h_{b f}\right)+\frac{\tau_{s, b-w}\left(h+2 h_{b f}\right)^{2}}{6 \eta}
$$

The power loss on the channel per unit channel width is:

$$
P O W_{\text {conv }}=\Delta p q_{v, \text { conv }}=\frac{2 \tau_{s, b-w} \Delta l}{h+2 h_{b f}}\left[\bar{u}\left(h+2 h_{b f}\right)+\frac{\tau_{s, b-w}\left(h+2 h_{b f}\right)^{2}}{6 \eta}\right]
$$

where $\Delta \mathrm{p}$ is the pressure drop on the channel. The critical power loss on the channel per unit channel width for starting the interfacial slippage is:

$$
P O W_{c r, \text { conv }}=\frac{\tau_{s, b-w}^{2} \Delta l\left(h+2 h_{b f}\right)}{3 \eta}
$$

According to Eqs.(8) and (9), the interfacial slipping velocity is then expressed as:

$$
\bar{u}=\frac{\Delta P O W_{c o n v}}{2 \tau_{s, b-w} \Delta l}
$$

where $\triangle P O W_{\text {conv }}=P O W_{\text {conv }}-P O W_{\text {cr,conv }}$.

The mass flow rate per unit channel width through the channel is then:

$$
q_{m, \text { conv }}=\rho q_{v, \text { conv }}=\rho\left[\frac{\Delta P O W_{c o n v}}{2 \tau_{s, b-w} \Delta l}\left(h+2 h_{b f}\right)+\frac{\tau_{s, b-w}\left(h+2 h_{b f}\right)^{2}}{6 \eta}\right]
$$

The ratio of the mass flow rate through the channel calculated from the multiscale analysis to that calculated from the conventional continuum flow theory is:

$$
r_{m}=\frac{2 \rho_{b f}^{e f f} q_{v, b f}+\rho q_{v, h f}}{q_{m, \text { conv }}}=\left(r_{b / h}+1\right) r_{v, h f}
$$

where

$r_{v, h f}=\frac{q_{v, h f}}{q_{v, \text { conv }}}=\frac{A \lambda_{b f} \frac{1-r_{0}}{2}+\left\{\frac{1}{12}-\frac{1}{C_{y}}\left\{\frac{F_{2} \lambda_{b f}^{2}}{6}-\frac{\lambda_{b f}}{1+\frac{\Delta x}{D}}\left[\frac{1}{2}+\lambda_{b f}-\frac{\left(q_{0}-q_{0}^{n}\right) \Delta_{n-2}}{\left(q_{0}^{n-1}-q_{0}^{n}\right) h}\right]\right\}\right\} \frac{1}{\frac{1}{2}+\frac{D}{h}(n-1)}}{A \lambda_{b f} \frac{1+2 \lambda_{b f}}{2}+\frac{\left(1+2 \lambda_{b f}\right)^{2}}{6}}$

\section{CALCULATION}

In the present calculation, it was taken that $D=0.5 \mathrm{~nm}, \Delta_{n-2} / \mathrm{D}=$ $\Delta x / \mathrm{D}=0.15$ and $\eta_{\text {line }, i} / \eta_{\text {line }, i+1}=q_{0}^{m} ;$ Here $m$ is positive.

It is formulated that (Zhang, 2014 and 2019):

$\mathrm{C}_{y}\left(H_{b f}\right)=\frac{\eta_{b f}^{e f f}\left(H_{b f}\right)}{\eta}=a_{0}+\frac{a_{1}}{H_{b f}}+\frac{a_{2}}{H_{b f}^{2}}$

where $H_{b f}=h_{b f} / h_{c r, b f}, h_{c r, b f}$ is a critical thickness, and $a_{0}, a_{1}$ and $a_{2}$ are respectively constant.

$C_{q}$ is expressed as (Zhang, 2014 and 2019):

$C_{q}\left(H_{b f}\right)=\frac{\rho_{b f}^{e f f}\left(H_{b f}\right)}{\rho}=m_{0}+m_{1} H_{b f}+m_{2} H_{b f}^{2}+m_{3} H_{b f}^{3}$

where $m_{0}, m_{1}, m_{2}$ and $m_{3}$ are respectively constant.

The weak, medium and strong fluid-wall interactions were respectively considered and they respectively have the $7 \mathrm{~nm}, 20 \mathrm{~nm}$ and $40 \mathrm{~nm}$ values for $h_{c r, b f}$ and the other rheological parameter values are shown in Tables 1 and 2.

The other parameter values for these three interactions are respectively:

Weak interaction: $m=0.5, n=3, q_{0}=1.03$

Medium interaction: $m=1.0, n=5, q_{0}=1.1$

Strong interaction: $m=1.5, n=8, q_{0}=1.2$
Table 1 Fluid viscosity data for different fluid-wall interactions (Zhang, 2014)

\begin{tabular}{|c|c|c|c|}
\hline Parameter & $a_{0}$ & $a_{1}$ & $a_{2}$ \\
\hline Strong & 1.8335 & -1.4252 & 0.5917 \\
\hline Medium & 1.0822 & -0.1758 & 0.0936 \\
\hline Weak & 0.9507 & 0.0492 & $1.6447 \mathrm{E}-4$ \\
\hline
\end{tabular}

Table 2 Fluid density data for different fluid-wall interactions (Zhang, 2014)

\begin{tabular}{|c|c|c|c|c|}
\hline Parameter & $m_{0}$ & $m_{1}$ & $m_{2}$ & $m_{3}$ \\
\hline Strong & 1.43 & -1.723 & 2.641 & -1.347 \\
\hline Medium & 1.30 & -1.065 & 1.336 & -0.571 \\
\hline Weak & 1.116 & -0.328 & 0.253 & -0.041 \\
\hline
\end{tabular}

\section{RESULTS}

\subsection{Value of $\boldsymbol{r}_{b / h}$}

Figure 2(a) shows that when the fluid-wall interaction is weak, for $\lambda_{b f} \leq 0.02$, the value of $r_{b / h}$ is normally no more than $5 \%$ whenever the interfacial slippage occurs or not $(A=0$ represents the case of no interfacial slippage); For this case, the contribution of the adsorbed layer to the flow is negligible. For a given $\lambda_{b f}$, the increase of $A$ i.e. the increase of the interfacial slippage significantly increases the value of $r_{b / h}$; This indicates that the interfacial slippage enlarges the proportion of the adsorbed layer flow in the total flow. It is due to the velocity profile modification across the channel height by the interfacial slippage. It is shown that when the thickness of the adsorbed layer is on the same scale with the continuum fluid film thickness, for a significant interfacial slippage (i.e. for $A>1$ ), the flow rate of the adsorbed layer is also on the same scale with the flow rate of the intermediate continuum fluid. For this case, a multiscale analysis may be required to accurately calculate the total flow rate through the channel by incorporating the adsorbed layer flow.

Figures 2(a-c) show that for a given $\lambda_{b f}$ and for the case of no interfacial slippage (i.e. $A=0$ ), the strengthening of the fluid-wall interaction significantly reduces the value of $r_{b / h}$; When the fluid-wall interaction is strong, the adsorbed layer is almost immobile and its contribution to the flow is negligible even when $\lambda_{b f}$ reaches 0.4 . However, when the interfacial slippage occurs, for the same $\lambda_{b f}$ and $A$, the fluid-wall interaction appears to have a weak influence on the value of $r_{b / h}$. In the case of the interfacial slippage such as for $\mathrm{A} \geq 1$, even for the strong fluid-wall interaction, when the thickness of the adsorbed layer is on the same scale with the continuum fluid film thickness (i.e. $\left.\lambda_{b f} \geq 0.1\right)$, the flow rate of the adsorbed layer is also on the same scale with the flow rate of the continuum fluid (i.e. $r_{b / h} \geq 0.1$ ). For this case, a multiscale analysis is appropriate for calculating the flow rate through the channel by incorporating the adsorbed layer flow.

Figures 2(b) and (c) strongly indicate that when the fluid-wall interaction is medium or strong, generating the fluid-wall interfacial slippage is a very effective way for significantly improving the mass transfer rate through the channel. For a narrow channel such as $\lambda_{b f} \geq$ 0.25 , when the interfacial slippage is so large as $A$ reaches 100 , the flow rate of the adsorbed layer can be more than half of the continuum fluid flow rate. Figure 2 (c) shows that when $\lambda_{b f} \geq 0.43$ and $A \geq 10$, the 
flow rate of the adsorbed layer is even greater than that of the intermediate continuum fluid, when the fluid-wall interaction is strong. Nevertheless, for a strong fluid-wall interaction, generating the interfacial slippage may require a much greater power loss on the channel as indicated by the presented equations (Zhang,2019), which yields the corresponding pressure gradient for the interfacial slippage as shown by Eq.(3). It should be attended that for a highly strong fluid-wall interaction, the interfacial slippage may first occur on the adsorbed layerfluid interface under a high pressure gradient.

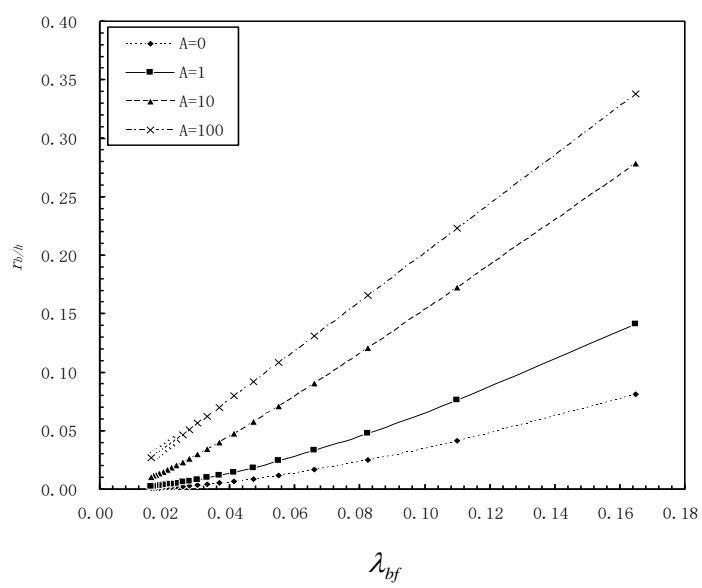

(a) For the weak fluid-wall interaction

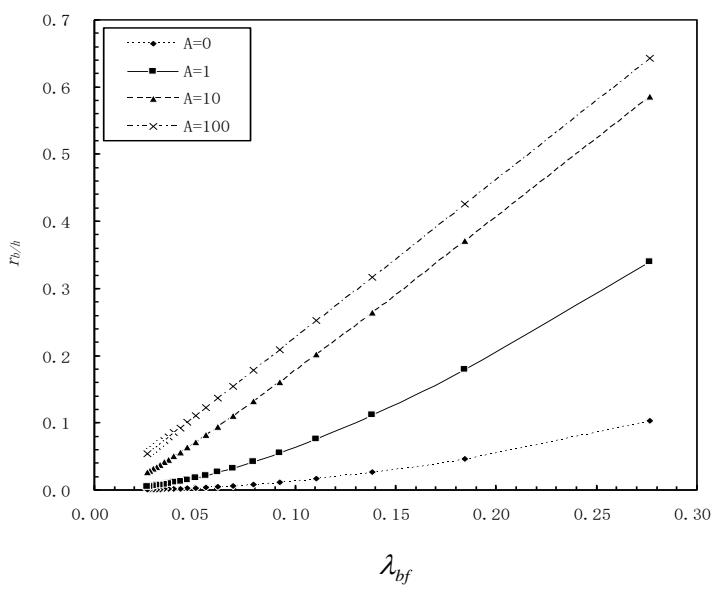

(b) For the medium fluid-wall interaction

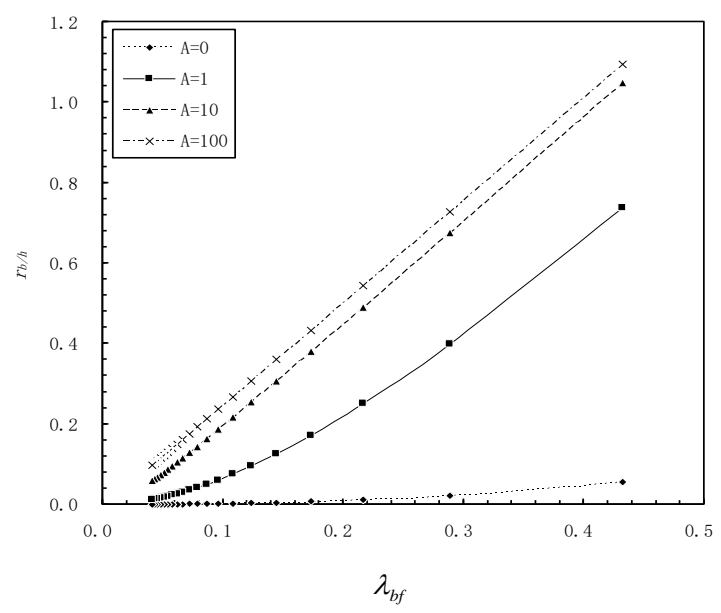

(c) For the strong fluid-wall interaction

Fig. 2 Values of $r_{b / h}$.

\subsection{Value of $r_{m}$}

Figure 3(a) shows the values of $r_{m}$ for different $\lambda_{b f}$ and $A$ when the fluid-wall interaction is weak. The values of $r_{m}$ are overall more than $90 \%$ when $\lambda_{b f} \leq 0.16$ and $A \leq 100$. It indicates that for a weak fluidwall interaction, the flow rate through the channel calculated from the conventional continuum flow theory is close to that calculated from the multiscale calculation when $\lambda_{b f} \leq 0.16$ and $A \leq 100$. The overestimation of the conventional continuum flow theory is due to the adsorbed layer effect, which reduces the flow velocity a little. For the weak fluid-wall interaction, the value of $r_{m}$ is lower for a higher $\lambda_{b f}$ or/and a greater value of $A$ (i.e. an increased interfacial slippage); For $\lambda_{b f} \leq 0.02$, the flow rate through the channel appears normally predictable from the conventional continuum flow theory whenever the interfacial slippage occurs or not.

Figure 3(b) shows the values of $r_{m}$ for different $\lambda_{b f}$ and $A$ when the fluid-wall interaction is medium-level. In this case, for $\lambda_{b f} \leq 0.02$, the conventional continuum flow theory calculation appears close to the multiscale calculation as $r_{m}>0.9$. For this case, the conventional calculation may be acceptable by ignoring the existence of the adsorbed layer, whenever the interfacial slippage occurs or not. However, when the thickness of the adsorbed layer is on the same scale with the continuum fluid film thickness i.e. $\lambda_{b f} \geq 0.1$, the values of $r_{m}$ are considerably lower than unity for $A \leq 10$. For these cases, it is not allowable to ignore the existence of the adsorbed layer and a multiscale calculation is required by treating the adsorbed layer as a flowing layer. However, when the interfacial slippage is so large as $A \geq 100$, the values of $r_{m}$ are close to unity in the entire range of $\lambda_{b f}$. It indicates that for a large interfacial slippage, the conventional continuum flow theory is sufficient for calculating the flow rate through the channel when the fluid-wall interaction is medium-level.

Figure 3(c) shows the similar results of $r_{m}$ as in Fig.3(b) when the fluid-wall interaction is strong. For a strong fluid-wall interaction, when $\lambda_{b f} \leq 0.02$, the conventional calculation may be sufficient by treating the adsorbed layer as an immobile solid layer adhering to the wall surface whenever the interfacial slippage occurs or not; When $\lambda_{b f} \geq 0.1$ and $A \leq 10$, a multiscale calculation is required by treating the adsorbed layer as a flowing layer; However, when the interfacial slippage is large as $A \geq 100$, the conventional calculation can be implemented across the whole channel height for the entire range of $\lambda_{b f}$ as the values of $r_{m}$ are close to unity.

\section{CONCLUSIONS}

The effect of the adsorbed layer in a micro/nano channel flow is investigated by a multiscale analysis. The channel flow is driven by the pressure and consists of the adsorbed layer flow and the intermediate continuum fluid flow. The interfacial slippage can occur on the adsorbed layer-wall surface interface depending on the operating condition, while it is absent elsewhere. The following conclusions are obtained:

(1) When the ratio $\lambda_{b f}$ of the thickness of the adsorbed layer to the film thickness of the intermediate continuum fluid is no more than 0.02 , the effect of the adsorbed layer is negligible and the continuum flow theory can calculate the flow rate through the channel by ignoring the existence of the adsorbed layer in spite of both the fluid-wall interaction and the interfacial slippage occurrence.

(2) The interfacial slippage enlarges the proportion of the adsorbed layer flow in the total flow through the channel. In spite of the fluidwall interaction, when the thickness of the adsorbed layer is on the same scale with the continuum fluid film thickness, for a significant interfacial slippage (i.e. for $\mathrm{A}>1$ ), the flow rate of the adsorbed layer is also on the same scale with the flow rate of the intermediate continuum fluid. For this case, a multiscale analysis can accurately calculate the total flow rate through the channel by incorporating the adsorbed layer flow. However, for the medium or strong fluidwall interactions, when the interfacial slippage is so large as $\mathrm{A} \geqslant$ 
100 , the continuum flow theory can be implemented across the whole channel height for the entire range of $\lambda_{b f}$ for calculating the total mass flow rate through the channel.

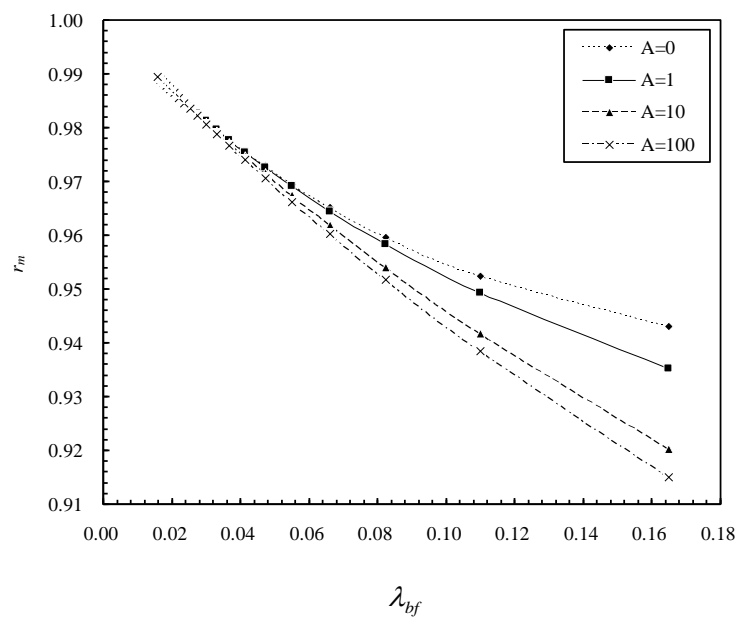

(a) For the weak fluid-wall interaction

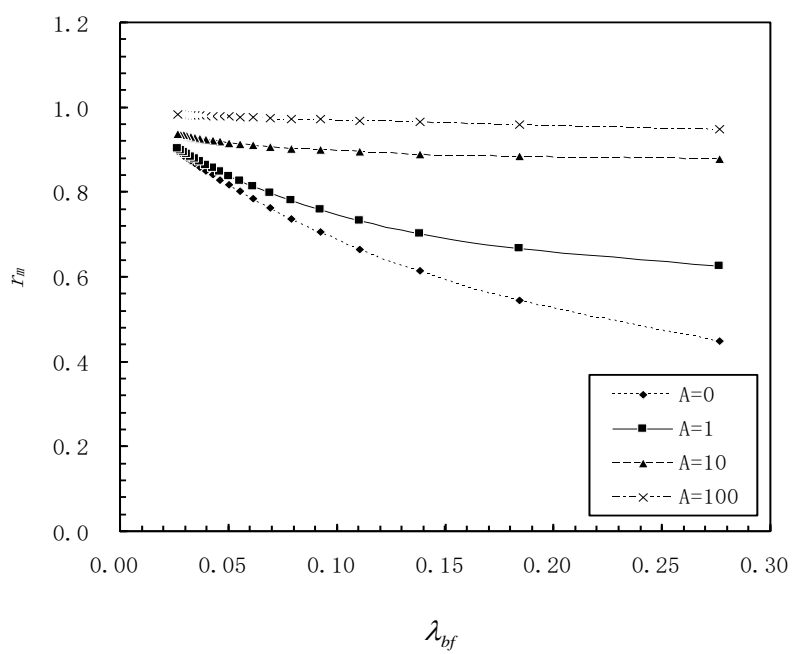

(b) For the medium fluid-wall interaction

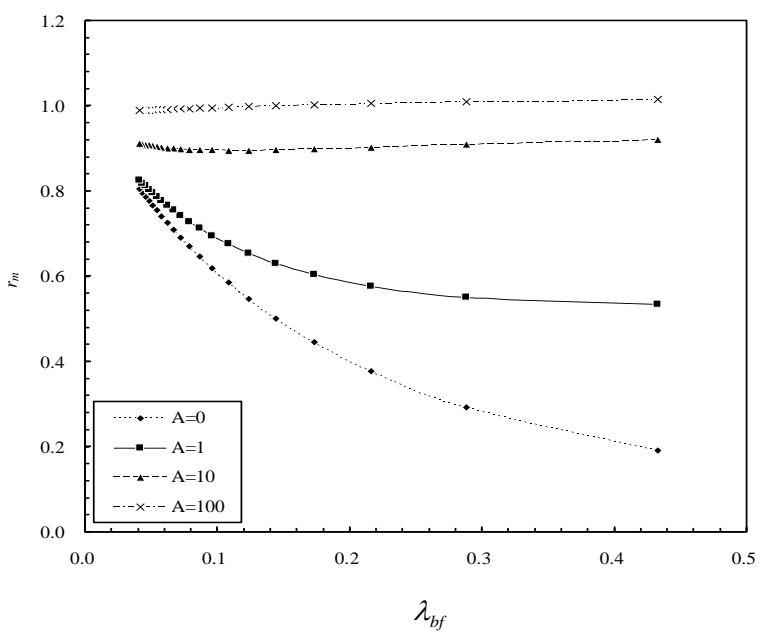

(c) For the strong fluid-wall interaction

Fig. 3 Values of $r_{m}$

(3) When the fluid-wall interaction is medium or strong, generating the fluid-wall interfacial slippage is very efficient for improving the mass transfer rate through the channel.

\section{REFERENCES}

Atkas, O., Aluru, N. R., 2002, "A combined continuum/DSMC technique for multiscale analysis of microfluidic filters." J. Comput. Phys, 178,342-372.

https://doi.org/10.1006/jcph.2002.7030

Cho, Y. I., 1982, "Non-Newtonian fluids in circular pipe flow." $A d v$. Heat Transf., 15, 59-141. https://doi.org/10.1016/S0065-2717(08)70173-4

Gee, M. L., McGuiggan, P. M., Israelachvilli, J. N., 1990, "Liquid to solidlike transitions of molecularly thin films under shear." J. Chem. Phys., 93, 1895.

https://doi.org/10.1063/1.459067

Heinbuch, U., Fischer, J., 1989, "Liquid flow in pores: Slip, no-slip, or multilayer sticking." Phys. Rev. A, 40, 1144. https://doi.org/10.1103/PhysRevA.40.1144

Jabbarzadeh, A., Atkinson, J. D., Tanner, R. I.,1997, "Rheological properties of thin liquid films by molecular dynamics simulations." J. Non-Newtonian Fluid Mech., 69, 169-193. http://dx.doi.org/10.1016//S0377-0257(96)01520-0

Joseph, P., Cottin-Bizonne, C., Benoît, J. M., Ybert, C., Journet, C., Tabeling, P., Bocquet, L., 2006, "Slippage of water past superhydrophobic carbon nanotube forests in microchannels." Phys. Rev. Lett., 97, 156104.

https:Ildoi.org/10.1103/PhysRevLett.97.156104

Kawamura, T., Takami, H., Kuwahara, K., 1986, “Computation of high Reynolds number flow around a circular cylinder with surface roughness." Fluid Dyn. Res., 1, 145-150.

https:Ildoi.org/10.1016/0169-5983 (86)90014-6

Lemenand, T., Peerhossaini, H., 2002, "A thermal mode for prediction of the Nusselt number in a pipe with chaotic flow." Appl. Therm. Eng., 22, 1717-1730. $\underline{\text { https://doi.org/10.1016/s1359-4311(02)00075-3 }}$

Liu, J., Chen, S., Nie, X., Robbins, M. O., 2007, “A continuum-atomistic simulation of heat transfer in micro- and nano- flows." J. Comput. Phys., 227, 279-291.

http://dx.doi.org/10.1016/i.jcp.2007.07.014

Nie, X. B., Chen, S., Robbins, M. O., 2004, “A continuum and molecular dynamics hybrid method for micro- and nano- fluid flow." J. Fluid Mech., 500, 55-64. http://dx.doi.org/10.1017/s0022112003007225

Sun, J., He, Y., Tao, W. Q., 2010, "Scale effect on flow and thermal boundaries in micro-/nano- channel flow using molecular dynamicscontinuum hybrid simulation method." Int. J. Num. Meth. Eng., 81, 207228. http://dx.doi.org/10.1002/nme.2683

Thompson, P. A., Robbins, M. O., 1990, "Shear flow near solids: Epitaxial order and flow boundary conditions." Phys. Rev. A, 41, 6830. https://doi.org/10.1103/PhysRevA.41.683

Vallikivi, M., Hultmark, M., Bailey, S. C. C., Smits, A. J., 2011, "Turbulence measurements in pipe flow using a nanoscale thermal anemometry probe." Exp. Fluids, 51, 1521-1527. https:Ildoi.org/10.1007/s00348-011-1165-4 
Yang, X., Zheng, Z. C., 2010, "Effects of channel scale on slip length of flow in micro/nano channels." ASME J. Fluids Eng., 132, 061201.

http://dx.doi.org/10.1115/1.4001619

Yen, T. H., Soong, C. Y., Tzeng, P. Y., 2007, "Hybrid molecular dynamics-continuum simulation for nano/mesoscale channel flows." Microfluid. Nanofluid., 3, 665-675.

http://dx.doi.org/10.1007/s10404-007-0154-7

Zhang, Y. B., 2014, "Lubrication analysis for a line contact covering from boundary lubrication to hydrodynamic lubrication: Part I- Micro contact results." J. Comput. Theor. Nanosci., 11, 62-70.

https: Ildoi.org/10.1166/jctn.2014.3336
Zhang, Y. B., 2016, "The flow equation for a nanoscale fluid flow." Int.

J. Heat Mass Transf., 92, 1004-1008.

https://doi.org/10.1016/j.ijheatmasstransfer.2015.09.008

Zhang, Y. B., 2019, "Power losss in multiscale mass transfer." Front. Heat Mass Transf., 13, 22.

http://dx.doi.org/10.5098/hmt.13.22

Zhang, Y. B., 2020, "Modeling of flow in a very small surface separation.” Appl. Math. Mod., 82, 573-586.

https://doi.org/10.1016/j.apm.2020.01.069. 